\title{
ARTICLE
}

Received 27 Jun 2014 | Accepted 17 Aug 2014 | Published 25 Sep 2014 DOI: 10.1038/ncomms6008

\section{Tenfold increase in the lifetime of blue phosphorescent organic light-emitting diodes}

Yifan Zhang ${ }^{1}$, Jaesang Lee $^{2} \&$ Stephen R. Forrest ${ }^{1,2,3}$

Organic light-emitting diodes are a major driving force of the current information display revolution due to their low power consumption and potentially long operational lifetime. Although electrophosphorescent organic emitters have significantly lower power consumption than fluorescent emitters, the short lifetime of electrophosphorescent blue devices has prevented their application in displays for more than a decade. Here, we demonstrate a novel blue electrophosphorescent device with a graded dopant concentration profile in a broadened emissive layer, leading to a lower exciton density compared with a conventional device. Thus, triplet-polaron annihilation that leads to long-term luminescent degradation is suppressed, resulting in a more than threefold lifetime improvement. When this strategy is applied to a two-unit stacked device, we demonstrate a lifetime of $616 \pm 10 \mathrm{~h}$ (time to $80 \%$ of the $1,000 \mathrm{cdm}^{-2}$ initial luminance) with chromaticity coordinates of $[0.15,0.29]$, representing a tenfold lifetime improvement over a conventional blue electrophosphorescent device.

\footnotetext{
${ }^{1}$ Department of Physics, University of Michigan, 2238 EECS, Ann Arbor, Michigan 48109, USA. ${ }^{2}$ Department of Electrical Engineering and Computer Science, University of Michigan, 2238 EECS, Ann Arbor, Michigan 48109, USA. ${ }^{3}$ Department of Materials Science and Engineering, University of Michigan, Ann Arbor, Michigan 48109, USA. Correspondence and requests for materials should be addressed to S.R.F. (email: stevefor@umich.edu).
} 
O rganic light-emitting diodes ${ }^{1}$ (OLEDs) are current-driven electroluminescent devices that have been proven successful in enabling a range of applications, including mobile displays, televisions and lighting ${ }^{2-4}$. Following electronhole recombination, two types of molecular excited states (called excitons), singlets and triplets, are formed with a ratio of 1:3 determined by quantum spin statistics ${ }^{5}$. Fluorescent OLEDs employ spin-antisymmetric singlets for emission, and thus have the internal quantum efficiency (IQE) limit of $25 \%$. Although triplet-triplet annihilation can be used to improve the IQE of fluorescent OLEDs beyond this limit ${ }^{6,7}$, their efficiencies are far inferior to the routinely realized $100 \% \mathrm{IQE}^{8}$ in electrophosphorescent OLEDs (PHOLEDs) ${ }^{9}$ that use both singlets and spin-symmetric triplet states for emission.

Besides high efficiency, long operational lifetime is essential for a technology to achieve commercial success. After more than a decade of research, significant breakthroughs in the lifetime of green and red PHOLEDs have been achieved, with reported T50 (time to $50 \%$ of the initial luminance $L_{0}=1,000 \mathrm{~cd} \mathrm{~m}^{-2}$ ) as long as $10^{6} \mathrm{~h}^{10-13}$. Even longer lifetimes have been reported at manufacturers' websites (see, for example, www.udcoled.com). In contrast, surprisingly little progress has been made in the improvement of blue PHOLED lifetime. For example, a T50 of only several hours for $L_{0}=1,000 \mathrm{~cd} \mathrm{~m}^{-2}$ has been reported for a PHOLED with the blue emitting iridium (III) FIrpic (bis[(4,6difluorophenyl)-pyridinato- $\mathrm{N}, \mathrm{C}^{2}$ ] picolinate $)^{14}$ as the emitter ${ }^{15}$. As a result, relatively inefficient blue fluorescent OLEDs remain dominant in OLED displays, making the blue pixels a bottleneck to improvements in display performance.

It has been suggested that the intrinsic degradation of blue PHOLEDs results from energetically driven formation of traps ${ }^{16,17}$ that can quench excitons and also act as non-radiative charge recombination centres. These traps are formed due to bimolecular triplet-polaron annihilation (TPA) where energy is transferred from triplets to polarons (that is, charged molecules). This, in turn, results in a high-energy polaron (approximately twice the triplet energy) that, on thermalization, can break bonds on the molecule on which it resides. Owing to their high triplet energy, excess energy dissipated through TPA in blue PHOLEDs is significantly higher than for red or green PHOLEDs, explaining the faster degradation in the former case. Furthermore, although blue fluorescent devices are by no means immune to this degradation path, their lower triplet energies lead to lower energy dissipation, and thus less destructive TPA events.

Since these fundamental mechanisms were first identified, to our knowledge there has been no direct evidence that blue PHOLED lifetime can be improved by reducing TPA in a practical, high-efficiency device. One potential route to reduce degradation suggested by this analysis is to decrease the exciton density in the emissive layer (EML) and consequently reduce the occurrence of TPA. Recently, Erickson and Holmes ${ }^{18}$ have demonstrated increased efficiency by the extension of the exciton formation zone in a green PHOLED from $15 \mathrm{~nm}$ to $>80 \mathrm{~nm}$ through

graded mixing of electron and hole-transporting host molecules. No lifetime data were reported in that work. In addition, the use of a non-uniform dopant profile in OLEDs was previously reported ${ }^{19-21}$ to improve efficiency; however, it is unclear whether the PHOLED exciton formation zone was also broadened as a consequence of the profiles employed, and its consequences on device lifetime were not explored.

Here we show that grading the blue dopant in the EML of a PHOLED significantly extends the lifetime of the device by extending the exciton formation zone. The broadening of the exciton distribution, and hence the device lifetime, is further increased by using a sufficiently high concentration of a dopant whose highest occupied molecular orbital level (HOMO) is chosen above that of the host to allow for holes to conduct directly on the dopant. To our knowledge, this is the first report of an OLED with a graded doping profile that extends the distribution of excitons within the EML, thus significantly having an impact on device operational lifetime. Furthermore, engineering the dopant and host frontier energy levels in addition to concentration grading provides a powerful tool that can be used to develop materials systems and device architectures to extend device lifetime even beyond that reported here.

\section{Results}

PHOLED device structure and characteristics. Figure 1a shows the chemical structure of the host 4,4'-bis(3-methylcarbazol-9yl)-2,2'-biphenyl (mCBP) and blue dopant iridium (III) tris[3(2,6-dimethylphenyl)-7-methylimidazo[1,2-f] phenanthridine] $\left(\operatorname{Ir}(\mathrm{dmp})_{3}\right)^{17}$. We compare five PHOLEDs shown in Fig. 1b, with their structural details provided in Methods. The hole transport layers (HTLs) and/or EMLs of the devices D1-D3 consist of a 20nm-thick layer of 4,4'-bis[N-(1-naphthyl)-N-phenyl-amino]biphenyl and a 30-nm-thick 13 vol\% $\operatorname{Ir}(\mathrm{dmp})_{3}$ doped in mCBP (Device D1), 50-nm-thick 13 vol\% $\operatorname{Ir}(\mathrm{dmp})_{3}$ doped mCBP (Device D2) and a $50-\mathrm{nm}$ layer of $\operatorname{Ir}(\mathrm{dmp})_{3}$ doped mCBP, with the doping concentration varying linearly with position (Device D3) from 18 vol\% (close to the anode) to 8 vol\% (close to the cathode). Devices D1S and D3S are two-unit stacked OLEDs whose HTL/EMLs are analogous to D1 and D3, respectively. Here, D1 adopts a conventional OLED structure almost identical to the blue PHOLED reported previously ${ }^{17}$ and serves as the control device.

The current density-voltage-luminance $(J-V-L)$, external quantum efficiency (EQE) and emission spectral characteristics of the five devices are shown in Fig. 2 and are summarized in Table 1. Replacing the HTL in D1 with an EML in D2 results in an increase in the operating voltage, as expected. Moreover, the use of a graded doping profile in the EML (D3) decreases the voltage compared with the uniformly doped D2. The EQE of D1 and $\mathrm{D} 2$ are almost identical above $1 \mathrm{~mA} \mathrm{~cm}^{-2}$, whereas the EQE of D3 is $>10 \%$ higher than those of D1 and D2. As expected ${ }^{22}$, D3S has approximately double the voltage and EQE compared with D3; for example, at $10 \mathrm{~mA} \mathrm{~cm}{ }^{-2}$, D3S operates at a voltage of $17.4 \mathrm{~V}$ and $\mathrm{EQE}=17.2 \%$ compared with $8.5 \mathrm{~V}$ and $9.3 \%$ for D3. Similar trends also exist between D1 and D1S. The spectra of D1, D2, D3 and D3S are almost identical, although the D1S spectrum shows a slightly higher vibronic peak energy at $\sim 500 \mathrm{~nm}$, due to optical microcavity effects ${ }^{23}$.

Charge transport and recombination. To understand the charge transport characteristics in the EML, we measured the ultraviolet photoelectron spectra of thin films of $\mathrm{mCBP}, \operatorname{Ir}(\mathrm{dmp})_{3}$, as well as $\operatorname{Ir}(\mathrm{dmp})_{3}$ deposited on mCBP (see Supplementary Figure 1). After considering the dipole energy shift when $\operatorname{Ir}(\mathrm{dmp})_{3}$ is deposited on $\mathrm{mCBP}$, then $\mathrm{mCBP}$ and $\operatorname{Ir}(\mathrm{dmp})_{3}$ have HOMO energies of 6.0 and $5.0 \mathrm{eV}$, respectively, as illustrated in Fig. 3a. Thus, it is expected that at sufficiently high concentrations, the dopant supports hole transport in the EML. The conduction mechanism is confirmed by the $J-V$ characteristics of hole-only devices that show improved hole transport when increasing the $\operatorname{Ir}(\mathrm{dmp})_{3}$ concentration in mCBP, as reported in Supplementary Figure 2. Also shown in Supplementary Figure 2 are the conduction characteristics of electron-only devices, which indicate electron transport in the EML occurs solely through $\mathrm{mCBP}$.

In this novel architecture, as illustrated in Fig. 3a, recombination occurs between an electron in the lowest unoccupied molecular orbital of mCBP and a hole in the $\operatorname{Ir}(\mathrm{dmp})_{3} \mathrm{HOMO}$, 


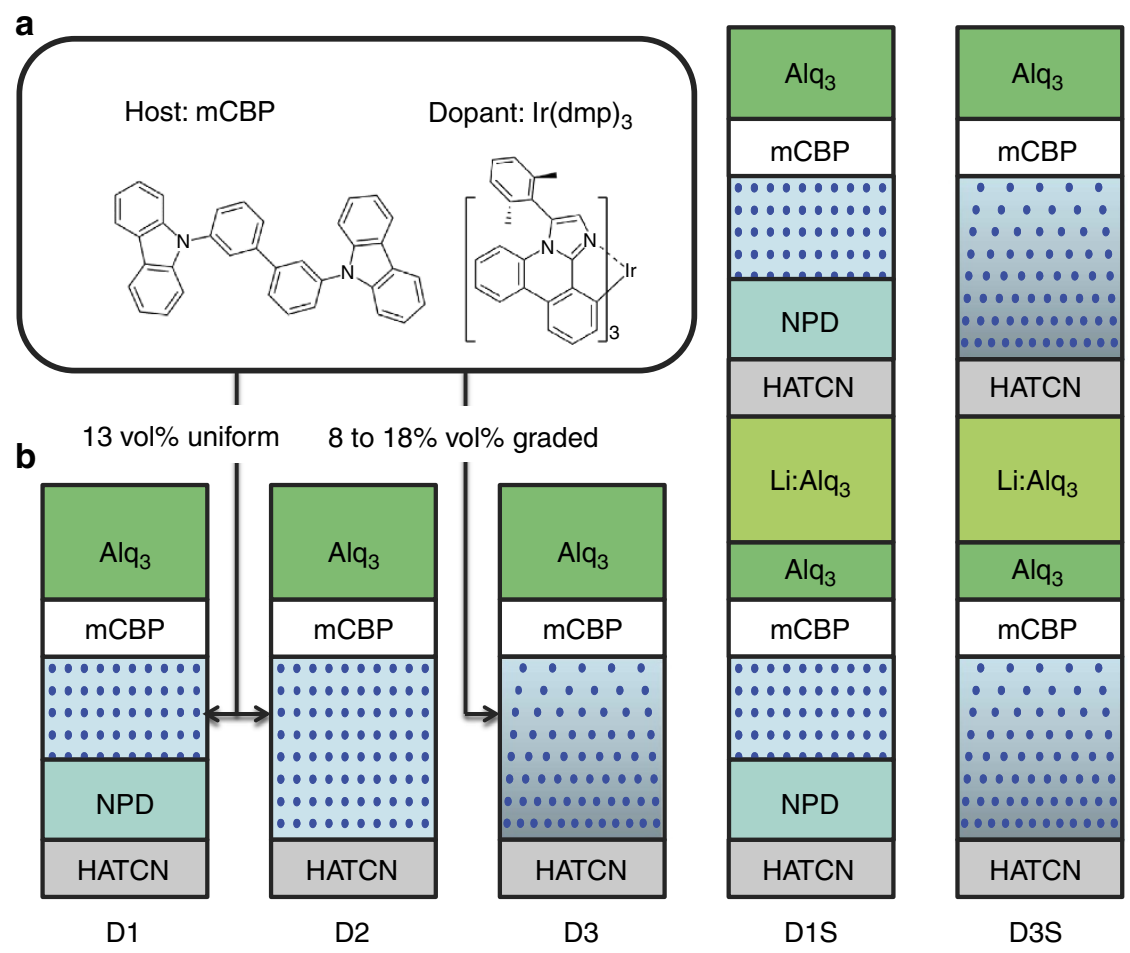

Figure 1 | Structures of the blue PHOLEDs. (a) Chemical structures of the host mCBP and phosphorescent dopant $\operatorname{Ir}(\mathrm{dmp})_{3}$ used in the EMLs of the blue PHOLEDs. (b) PHOLED device structures. HATCN, NPD and $\mathrm{Alq}_{3}$ are used for the HIL, HTL and electron transport layer (ETL) materials, respectively. The EMLs of both D1 and D2 employ a 13 vol\% doping concentration, and D2 replaces the HTL in D1 with an EML. D3 has an EML with the doping concentration graded from 18 to 8 vol\% with a maximum at the EML/HIL interface. Further, D1S and D3S are two-unit stacked OLEDs with 2 vol\% Li-doped $\mathrm{Alq}_{3}$ and HATCN as the interconnecting layers.

resulting in an exciplex. As the PHOLED emission is solely from the phosphorescence of $\operatorname{Ir}(\mathrm{dmp})_{3}$, the intermediate exciplex state rapidly transfers its energy to the $\operatorname{Ir}(\mathrm{dmp})_{3}$ triplet. Note that the thermalization loss when an electron on $\mathrm{mCBP}$ recombines with a hole in the $\operatorname{Ir}(\mathrm{dmp})_{3} \mathrm{HOMO}$ is $1.0 \mathrm{eV}$ less than for a hole in the mCBP HOMO, contributing to the relatively high operational stability of the blue PHOLEDs.

The hole conductivity in the graded EML of D3 increases as the distance to the hole injection layer (HIL)/EML interface is decreased due to the increasing concentration of $\operatorname{Ir}(\mathrm{dmp})_{3}$. In the opposite direction towards the EML/hole-blocking layer (HBL) interface, the hole conductivity decreases while the electron conductivity stays approximately constant. Thus, in contrast to the abrupt hole blocking by the EML/HBL interface in D1 and $\mathrm{D} 2$, in $\mathrm{D} 3$ the holes are gradually blocked by the hole conductivity gradient, leading to a simplified device architecture where the HTL is replaced by a high concentration of the hole-conducting phosphorescent dopant near the HIL. As a consequence, exciton formation in D3 occurs over an extended distance compared with either D1 or D2.

Exciton distribution in the emission zone. To determine the hole-blocking efficiency and thus the shape of the exciton formation zones in D1-D3, we fabricated a series of PHOLEDs with a thin, red-emitting 'sensing' layer using the red dopant, iridium (III) bis(2-phenyl quinolyl- $\left.\mathrm{N}, \mathrm{C}^{2^{\prime}}\right)$ acetylacetonate (PQIr) whose relative emission intensity can provide information about the spatial distribution of excitons in the EML (see Methods). Here, PQIr is co-doped at $2 \mathrm{vol} \%$ at different positions separated by $5 \mathrm{~nm}$ in the EMLs of D1-D3, with a doping layer width of $1.5 \mathrm{~nm}$. The HOMO and lowest unoccupied molecular orbital energies of PQIr are at 5.0 and $2.7 \mathrm{eV}$ relative to the vacuum level, respectively ${ }^{24}$. Owing to the low doping concentration and narrowness of the sensing layers, their presence should not significantly affect the charge transport or recombination properties in the EML. This is confirmed by the almost identical $J-V$ characteristics between devices with and without sensing layers (see Supplementary Figures 3, 4 and 5 for more details on the sensing layers).

From the emission spectra and EQE at $10 \mathrm{~mA} \mathrm{~cm}^{-2}$, as well as from the calculated light out-coupling efficiencies ${ }^{25}$ in the 'sensing' PHOLEDs (see Methods and Supplementary Note 3), we calculate the exciton densities versus position in the EMLs of D1-D3, with results in Fig. 3b. In D1 (that is, the conventional blue PHOLED), significant exciton accumulation occurs at the EML/HBL interface. In D2 that lacks an HTL, the exciton formation zone is broadened due to the increase in the EML thickness. As a result, the exciton density at the HIL/EML interface is reduced due to a correspondingly low concentration of holes. However, because of the reduced hole-transport efficiency, electrons penetrate deep into the EML, resulting in a peak exciton density near the HIL/EML interface. In contrast, efficient hole transport near the HIL/EML interface and the gradual hole blocking in the EML in D3 lead to a more uniform exciton distribution (and hence higher EQE) compared with both D1 and D2, with a peak density near to the centre of the EML. As the EMLs of D1 and D1S are identical, and D3 and D3S are also identical, Fig. $3 \mathrm{~b}$ also shows exciton density profiles of D1S and D3S, estimated at $50 \%$ of the exciton density in D1 and D3 due to the almost doubled EQEs.

PHOLED lifetime: experiment and model. Figure 4 shows the time evolution of the luminance, $L$, and the change in voltage from its initial value, $\Delta V=|V(t=0)-V(t)|$, for D1, D3 and D3S 


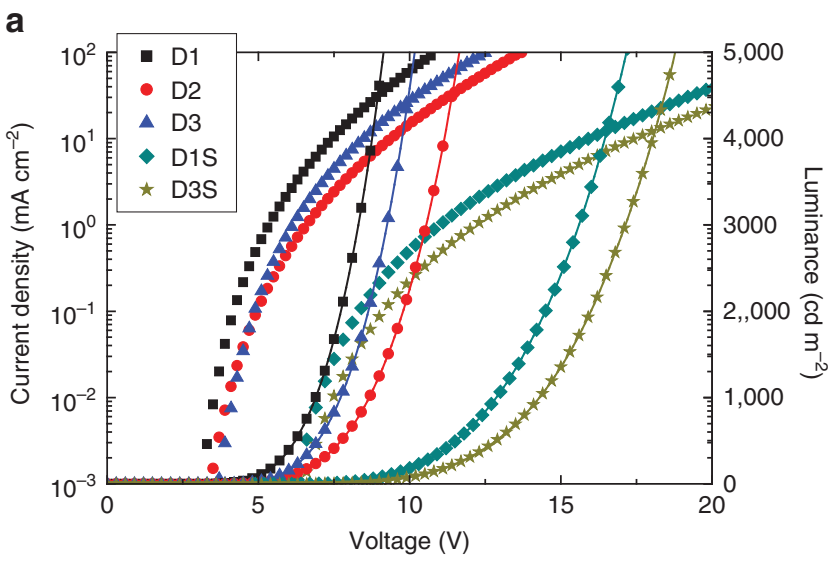

b

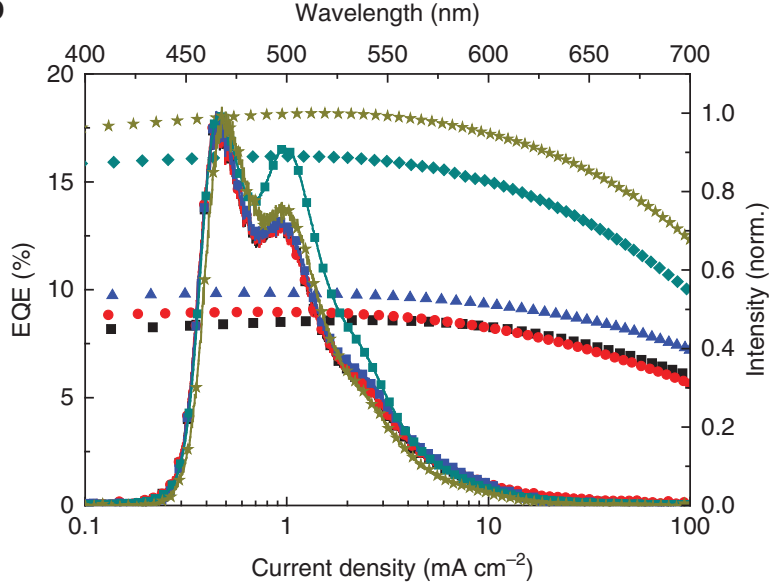

Figure 2 | Performance characteristics of the blue PHOLEDs. (a) Current density-voltage ( $J-V$, scattered markers) and luminance-voltage ( $L-V$, lineconnected markers) characteristics. (b) EQE versus $J$ (left axis) and emission spectra (right axis).

tested at room temperature and a constant current density for two initial luminances: $L_{0}=1,000$ and $3,000 \mathrm{~cd} \mathrm{~m}^{-2}$. In addition, we show these same characteristics for D2 and D1S tested at $L_{0}=3,000 \mathrm{~cd} \mathrm{~m}^{-2}$. The lifetimes show an increasing trend from D1 through D3, with $T 80=11.5,24.5$ and $39 \mathrm{~h}$, respectively, for D1-D3 at $L_{0}=3,000 \mathrm{~cd} \mathrm{~m}^{-2}$, consistent with the broadened exciton formation zone of the latter two devices (Fig. 3b). Note that $T 80$ for the control, D1, is consistent with similar results for this device reported previously ${ }^{6}$, and is only $\sim 29 \%$ that of the graded device, D3. Further, due to the reduced triplet density in the stacked OLEDs, T80 $=48$ and $106 \mathrm{~h}$ for D1S and D3S, respectively. At $L_{0}=1,000 \mathrm{~cd} \mathrm{~m}^{-2}, T 80=616 \pm 10 \mathrm{~h}$ for D3S, representing $>10$ times improvement from the previously investigated ${ }^{17}$ control, D1. The improvement in T50 for D3S is slightly less significant than T80, leading to an approximately sevenfold increase from D1.

To establish a quantitative relationship between the exciton density profiles and operational lifetimes, we model $L$ and $\Delta V$ as functions of time, $t$, using a model similar to Giebink et al. ${ }^{17}$ (see Methods). Briefly, the degradation of both $L$ and $V$ are attributed to the formation of traps in the EMLs due to TPA at a rate, $k_{\mathrm{Q}}$. The following trap bimolecular interactions are considered: trapelectron interactions at $\operatorname{rate}^{17}, k_{\mathrm{Qn}}=1.44 \times 10^{-13} \mathrm{~cm}^{3} \mathrm{~s}^{-1}$; trap-hole interactions at rate ${ }^{17}, k_{\mathrm{Qp}}=4.8 \times 10^{-14} \mathrm{~cm}^{3} \mathrm{~s}^{-1}$; and trap-triplet annihilation at rate, $k_{\mathrm{QN}}$. Trap formation depends on the local exciton density; hence, we model PHOLED degradation using the profiles in Fig. $3 \mathrm{~b}$. The model is fit to the degradation characteristics of all devices with $k_{\mathrm{Q}}$ and $k_{\mathrm{QN}}$ as free parameters (see Table 1); all other parameters are as previously determined for this materials combination ${ }^{17}$ (see Methods).

Yamamoto et al. $^{26}$ reported that water contamination of organic films during deposition leads to an accelerated initial degradation when the deposition background pressure is $>5 \times 10^{-8}$ Torr for PHOLEDs, almost identical to that of D1. In our case, organic films were deposited in a system with a base pressure of $5 \times 10^{-7}$ Torr; however, the TPA model ignores extrinsic effects such as water contamination. To account for these effects in our fits, $t=0$ corresponds to a normalized luminance of 0.95 and the initial value of $\Delta V$ was chosen to be non-zero (see Methods).

From the lifetime fit parameters summarized in Table $1, k_{\mathrm{Q}}$ and $k_{\mathrm{QN}}$ in all five devices are almost identical, indicating that the differences in their lifetimes are quantitatively explained by their different exciton densities at the same luminance. Compared with D1, the doubled lifetime in D2 is simply a result of changes in the exciton density profile, and the tripled lifetime in D3 is due to the increase in EQE (leading to a decrease of $J$ to achieve a given $L_{0}$ ), as well as to the significant spreading of the exciton formation zone. Here, the novel graded doping profile in a thick EML results in a threefold improvement in lifetime compared with the conventional uniform doping profile in a thin EML.

At 3,000 $\mathrm{cd} \mathrm{m}^{-2}$, the improvement in T80 from single element to stacked OLEDs is 4.1 -fold from D1 to D1S, and 2.7-fold from D3 to D3S. This is understandable from the decrease in $J$ (and thus triplet densities); at $3,000 \mathrm{~cd} \mathrm{~m}^{-2}, J$ of D1S is $42 \%$ of D1, and $J$ of D3S is $52 \%$ of D3. The more significant decrease in $J$ in D1S is due to the increase of its spectral peak at $500 \mathrm{~nm}$ (see Fig. 2b), which results in a higher luminance efficiency (in $\mathrm{cd} \mathrm{A}^{-1}$ ) at the same EQE. Thus, when combining the graded doping profile with a stacked structure, a tenfold improvement in T80 from D1 to D3S is observed.

Note that the TPA model considers degradation in the thin film bulk, but not at the interface ${ }^{27}$. Indeed, the low exciton density at the EML/HBL interface in D3 and D3S may also contribute to the observed increase in lifetime by reducing the rate of damage at this interface.

To estimate T50 at $L_{0}=1,000 \mathrm{~cd} \mathrm{~m}^{-2}$ for D3 and D3S, we extrapolate the TPA model fits. In addition, an empirical method often employed to model OLED degradation uses the adjusted exponential decay function: ${ }^{28} L(t)=L_{0} \exp \left[-(t / \tau)^{\beta}\right]$. Here, $\tau$ and $\beta$ are phenomenological parameters. This model also results in reasonable fits to the degradation data (Fig. 4c) and provides extrapolated values for T50 similar to those obtained from the physics-based TPA model (Table 1). From these fits, $T 50=3,500 \mathrm{~h}$ at $L_{0}=1,000 \mathrm{~cd} \mathrm{~m}^{-2}$ for D3S, which approaches that of blue fluorescent OLEDs with $T 50 \sim 10^{4} \mathrm{~h}$ (see, for example, www.idemitsu.com). Note that the emission of the devices reported here is light blue (although more saturated than the cyan colour of FIrpic). Colour tuning to achieve more saturated blue emission is commonly achieved in fluorescent blue display sub-pixels through the use of microcavities ${ }^{23,29}$ and/or colour filters. For example, a 70-nm-thick anode of indium-tin oxide (ITO) yields Commission Internationale d'Eclairage chromaticity coordinates of $[0.16,0.26]^{26}$, compared with $[0.16$, 0.31] for a 120-nm-thick ITO layer due to weak microcavity effects (see Supplementary Figure 6).

\section{Discussion}

Although the blue PHOLED lifetime reported here remains substantially less than that of red and green PHOLEDs at similar luminances, blue sub-pixels in displays operate at a considerably lower luminance than either the red or green sub-pixels. For 
Table 1 | Characteristics at $L_{0}=1,000 \mathrm{~cd} \mathrm{~m}^{-2}$ and lifetime fitting parameters for blue emitting PHOLEDs.*

\begin{tabular}{|c|c|c|c|c|c|c|c|c|c|c|}
\hline & \multicolumn{6}{|c|}{$L_{0}=1,000 \mathrm{~cd} \mathrm{~m}^{-2}$} & \multicolumn{2}{|c|}{ TPA model } & \multicolumn{2}{|c|}{ Empirical model } \\
\hline & $\mathbf{C I E}^{\dagger}$ & EQE (\%) & $J\left(\mathrm{~mA} \mathrm{~cm}^{-2}\right)$ & $V(V)$ & T80 (h) & T50 (h) & $k_{Q}\left(\mathrm{~cm}^{3} \mathrm{~s}^{-1}\right)$ & $k_{\mathrm{QN}}\left(\mathrm{cm}^{3} \mathrm{~s}^{-1}\right)$ & $\tau\left(10^{3} h\right)$ & $\beta$ \\
\hline D1 & {$[0.16,0.31]$} & $8.5 \pm 0.1$ & 6.2 & $6.9 \pm 0.2$ & $56 \pm 3$ & $510 \pm 15$ & $(1.0 \pm 0.1) \times 10^{-11}$ & $(7 \pm 1) \times 10^{-24}$ & $1.03 \pm 0.01$ & $0.54 \pm 0.01$ \\
\hline D2 & {$[0.16,0.31]$} & $8.5 \pm 0.2$ & 6.2 & $8.7 \pm 0.3$ & - & - & $(1.1 \pm 0.1) \times 10^{-11}$ & $(7 \pm 1) \times 10^{-24}$ & - & - \\
\hline D3 & {$[0.16,0.31]$} & $9.5 \pm 0.1$ & 5.7 & $7.7 \pm 0.2$ & $213 \pm 5$ & $1,500^{\ddagger} / 1600^{\S}$ & $(0.95 \pm 0.1) \times 10^{-11}$ & $(6 \pm 1) \times 10^{-24}$ & $2.96 \pm 0.04$ & $0.58 \pm 0.01$ \\
\hline D1S & {$[0.16,0.33]$} & $16.0 \pm 0.1$ & 2.8 & $12.9 \pm 0.1$ & - & - & $(0.96 \pm 0.1) \times 10^{-11}$ & $(7 \pm 1) \times 10^{-24}$ & - & - \\
\hline D3S & {$[0.15,0.29]$} & $18.0 \pm 0.2$ & 2.9 & $14.3 \pm 0.1$ & $616 \pm 10$ & $3,500^{\ddagger} / 3700^{\S}$ & $(0.9 \pm 0.1) \times 10^{-11}$ & $(7 \pm 1) \times 10^{-24}$ & $6.74 \pm 0.10$ & $0.63 \pm 0.01$ \\
\hline
\end{tabular}

EQE, external quantum efficiency; TPA, triplet-polaron annihilation;

*Errors for EQE and $V$ are s.d. from at least six devices, errors for $T 80$ and $T 50$ are s.d. from three devices and errors for the model parameters are the $95 \%$ confidence interval for fits. $\dagger$ Measured at $10 \mathrm{~mA} \mathrm{~cm}^{-2}$.

Estimated from extrapolations using the TPA model.

$\S$ Estimated from extrapolation using the adjusted exponential (empirical) model.
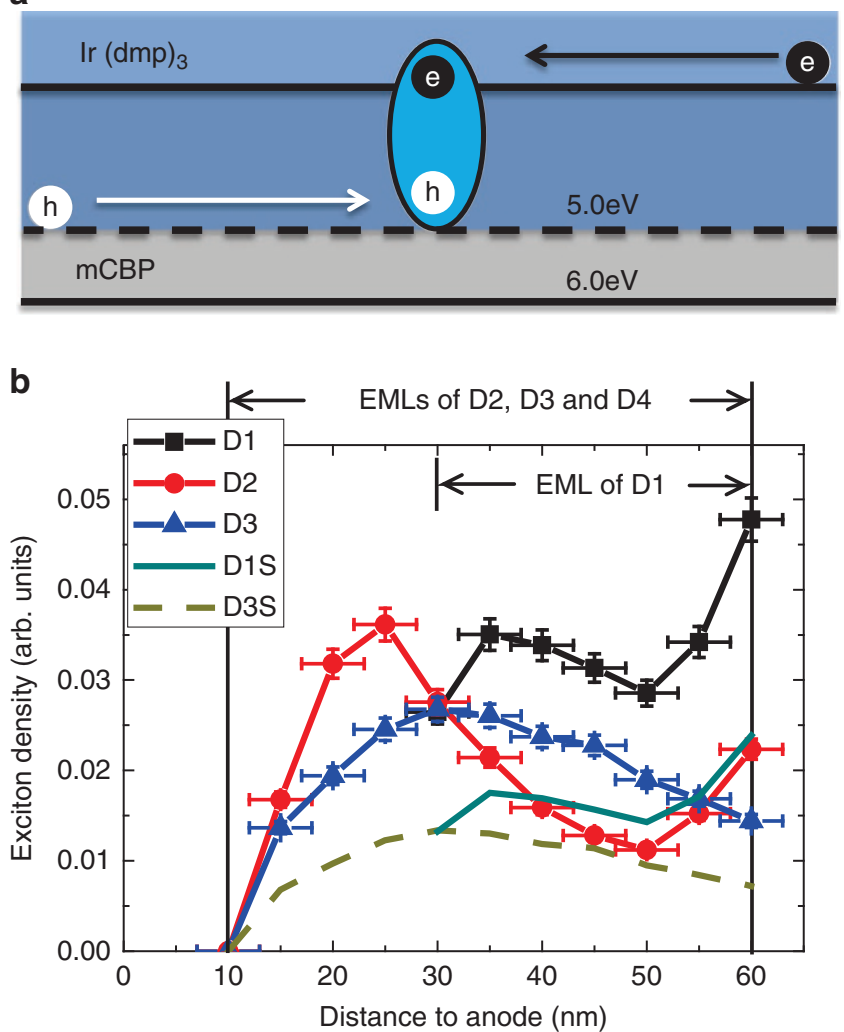

Figure 3 | Energetics and exciton density profiles in the PHOLED EML. (a) Electron and hole transport and recombination energetics in the blue PHOLED EMLs. (b) Exciton density profile in the EMLs of D1-D3 calculated from 'probe' devices employing PQIr in the red light-emitting sensing layer. The profiles are normalized to integrated exciton densities in the EMLs of unity. Moreover, the exciton density profiles of D1S and D3S are calculated from the D1 and D3 profiles, respectively. The errors in distance to anode arise from the determination of the Förster transfer radius for $\operatorname{Ir}(\mathrm{dmp})_{3}$ to PQIr of $\sim 3 \mathrm{~nm}$, and the errors in exciton density arise from the errors in EQEs (see Methods).

example, the required luminance to achieve an sRGB colour gamut $^{30}$ for green is 9.9 times the luminance for blue. Thus, a comparison between blue and green PHOLED lifetimes for displays suggests that the blue PHOLED sub-pixel luminance needs to be only $\sim 10 \%$ that of the green. Under such conditions (that is, $L_{0}=100 \mathrm{~cd} \mathrm{~m}^{-2}$ ), the TPA model estimates the blue PHOLED lifetime is $70,000 \mathrm{~h}$. Moreover, adopting a degradation acceleration factor that relates luminance to lifetime ${ }^{28}$, viz. $T 50\left(100 \mathrm{~cd} / \mathrm{m}^{2}\right)=T 50\left(1000 \mathrm{~cd} / \mathrm{m}^{2}\right) \times\left[\frac{1000 \mathrm{~cd} / \mathrm{m}^{2}}{100 \mathrm{~cd} / \mathrm{m}^{2}}\right]^{n}$ with $n=1.55$, the extrapolated blue PHOLED lifetime is $1.3 \times 10^{5} \mathrm{~h}$. These lifetimes approach the commercial green PHOLED lifetime $\left(10^{6} \mathrm{~h}\right)$ at $L_{0}=1,000 \mathrm{~cd} \mathrm{~m}^{-2}$.

In summary, we demonstrate a tenfold increase in blue PHOLED lifetime employing a stacked PHOLED structure with two EMLs having graded phosphorescent dopant concentrations. Grading used in combination with engineered frontier orbital energies of both the dopant and host materials significantly decreases the exciton density across the emission zone. This in turn increases the blue PHOLED external efficiency while reducing detrimental TPA that decreases device lifetime. Considering the different colour sub-pixel luminances used in displays, the improved blue PHOLED lifetimes approaches that of green PHOLEDs under normal operating conditions.

The novel device architecture employed is based on a fundamental physical understanding of the relationship between energy-driven TPA and device degradation, and hence should be generally applicable to a wide range of phosphorescent and fluorescent devices. Further lifetime improvements are anticipated by finding dopant/host combinations with conduction properties similar to the materials used here, and which minimize interactions between triplets on the dopant and polarons on the host molecules that lead to molecular decomposition.

\section{Methods}

Device fabrication. Thin films were deposited by thermal evaporation on pre-cleaned ITO-on-glass substrates in a system with a background pressure of $\sim 5 \times 10^{-7}$ Torr. Devices D1-D3 have the following structure: $120 \mathrm{~nm}$ ITO anode/ $10 \mathrm{~nm}$ hexaazatriphenylene hexacarbonitrile (HATCN) HIL/50 nm HTL + EML/ $5 \mathrm{~nm} \mathrm{mCBP} \mathrm{HBL} / 30 \mathrm{~nm}$ tris(8-hydroxyquinolinato)aluminum ( $\mathrm{Alq}_{3}$ ) electron transport layer/1.5 $\mathrm{nm}$ 8-hydroxyquinolinato lithium (Liq)/100 nm Al cathode. The structure of D1S and D3S is $120 \mathrm{~nm} \mathrm{ITO} / 10 \mathrm{~nm} \mathrm{HATCN} / 50 \mathrm{~nm} \mathrm{HTL}+\mathrm{EML} / 5 \mathrm{~nm}$ $\mathrm{mCBP} / 5 \mathrm{~nm} \mathrm{Alq} / 30 \mathrm{~nm} 2 \mathrm{vol} \%$ Li-doped $\mathrm{Alq}_{3} / 10 \mathrm{~nm}$ HATCN $/ 50 \mathrm{~nm} \mathrm{HTL}+$ $\mathrm{EML} / 5 \mathrm{~nm} \mathrm{mCBP} / 25 \mathrm{~nm} \mathrm{Alq} 3 / 1.5 \mathrm{~nm} \mathrm{Liq} / 100 \mathrm{~nm}$ Al. All devices were grown on pre-patterned ITO with an active area of $2 \mathrm{~mm}^{2}$ defined by openings in a polyimide layer that define the pixels. The PHOLEDs are packaged in an ultra-high purity $\mathrm{N}_{2}$-filled glove box with $<0.5$ p.p.m. oxygen and water concentration without exposure to air, following film deposition, by attaching a glass lid to the substrate using an epoxy seal around the perimeter. Devices for exciton sensing were grown on $70 \mathrm{~nm}$ of ITO with an active area of $0.785 \mathrm{~mm}^{2}$ defined by shadow masks during cathode deposition.

Device characterization. $J-V-L$ characteristics were measured using a parameter analyser (HP4145, Hewlett-Packard) and a calibrated photodiode (FDS1010-CAL from Thorlabs) following standard procedures ${ }^{31}$. Emission spectra were measured with a calibrated fibre-coupled spectrometer (USB4000, Ocean Optics) at $J=10 \mathrm{~mA} \mathrm{~cm}^{-2}$. Lifetime tests were carried out with PHOLEDs driven by constant current sources (U2722, Agilent), and the voltage and luminance are recorded by a data logger (Agilent 34972A). 
a

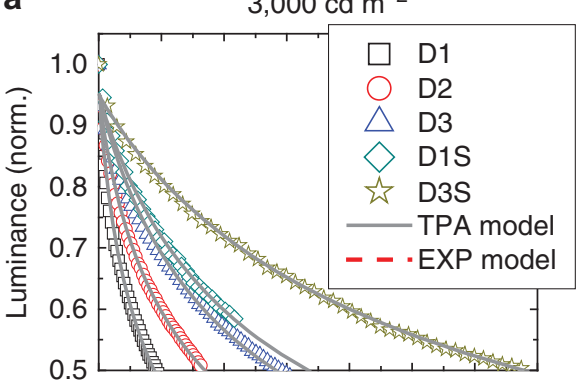

C

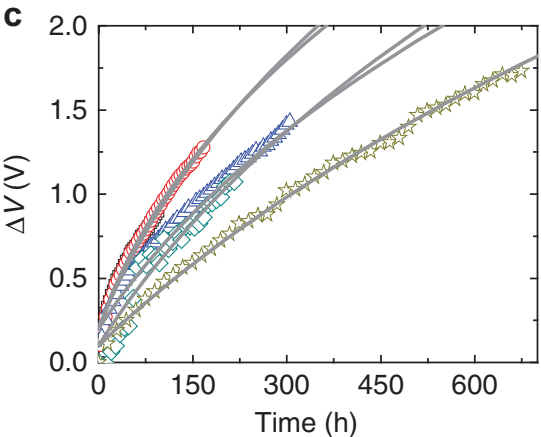

b

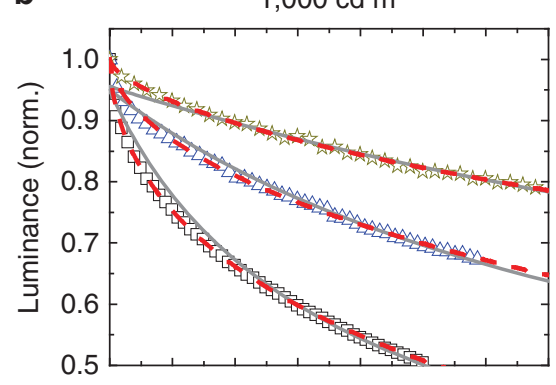

d

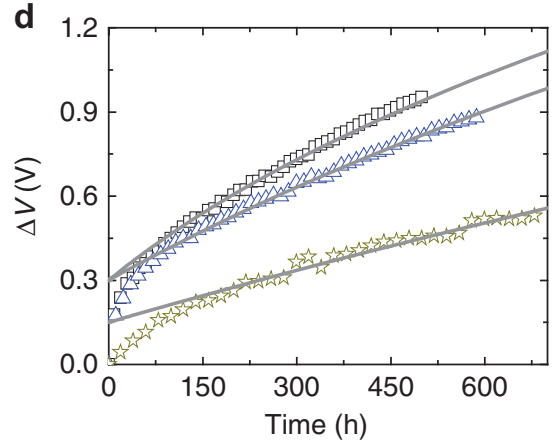

Figure 4 | Operational lifetime of the PHOLEDs. Time evolution of the normalized luminance, $L$, of blue PHOLEDs and change in operating voltage $\Delta V$ (offset to zero) at the initial luminance of $L_{0}=3,000 \mathrm{~cd} \mathrm{~m}^{-2}(\mathbf{a}, \mathbf{b})$ and $1,000 \mathrm{~cd} \mathrm{~m}^{-2}(\mathbf{c}, \mathbf{d})$. The data are fit to the TPA model using the exciton density profiles in Fig. 3b. The luminance for $L_{0}=1,000 \mathrm{~cd} \mathrm{~m}^{-2}$ (c) are also fit to the adjusted empirical exponential (EXP) model to estimate T50 (that is, degradation time leading to $50 \%$ decrease in $L$ ).

Exciton formation zone analysis. Local exciton densities $N(x)$ in the EMLs are calculated from the measured emission spectra, $\operatorname{EQE}(x)$ and the calculated outcoupling efficiency $\eta_{\mathrm{R}}(x)$ (at a wavelength of $595 \mathrm{~nm}$ corresponding to the peak in the PQIr emission spectrum), from PHOLEDs with PQIr sensing layer at position $x$ using:

$$
N(x)=A \times E Q E(x) \frac{N_{\mathrm{R}}^{\text {out }}(x)}{N_{\mathrm{R}}^{\text {out }}(x)+N_{\mathrm{B}}^{\text {out }}(x)} \frac{1}{\eta_{\mathrm{R}}(x)}
$$

Here, $A$ is a normalization factor such that $\int_{E M L} N(x) d x=1, N_{R}^{\text {out }}(x) / N_{B}^{\text {out }}(x)$ is the outcoupled photon number ratio from PQIr versus $\operatorname{Ir}(\mathrm{dmp})_{3}$, obtained from the ratio of red and blue peaks of the emission spectra.

Degradation model. The time evolution of $L(t)$ and $V(t)$ is modelled following Giebink et al. ${ }^{17}$ by assuming trap (with density $Q(x, t)$ ) formation due to TPA, and subsequent interactions with electrons with density $n(x, t)$, holes with density $p(x$, $t)$ and excitons with density $N(x, t)$ :

$$
\begin{aligned}
& G(x)-r n(x, t) p(x, t)-k_{Q n} Q(x, t) n(x, t)=0 \\
& G(x)-r n(x, t) p(x, t)-k_{Q p} Q(x, t) p(x, t)=0 \\
& r n(x, t) p(x, t)-\left(\frac{1}{\tau_{N}}+k_{Q T} Q(x, t)\right) N(x, t)=0
\end{aligned}
$$

Here $r=1.7 \times 10^{-13} \mathrm{~cm}^{3} \mathrm{~s}^{-1}$ is the Langevin recombination rate, $\tau_{\mathrm{N}}=1.1 \mu \mathrm{s}$ is the triplet lifetime and $G(x)$ is the local recombination rate, calculated from the local exciton density $N(x)$ using $G(x)=\frac{I}{e} \frac{N(x)}{\int N(x) d x}$, where $e$ is the electron charge. Current densities at $L_{0}=1,000 \mathrm{~cd} \mathrm{~m}^{-2}$ are provided in Table 1 and at $3,000 \mathrm{~cd} \mathrm{~m}^{-2}$ are $J=21,21,17.5,8.9$ and $9.1 \mathrm{~mA} \mathrm{~cm}^{-2}$ for D1, D2, D3, D1S and D3S, respectively. Trap formation is attributed to TPA using:

$$
\frac{d Q(x, t)}{d t}=k_{Q} N(x, t) n(x, t)
$$

Then

$$
\begin{aligned}
& L(t)=B \int N(x, t) \eta_{B}(x) d x \\
& \Delta V(t)=\Delta V(0)+\int \frac{e}{\varepsilon \varepsilon_{0}} x Q(x, t) d x
\end{aligned}
$$

where $B$ is a normalization factor, $\eta_{\mathrm{B}}(x)$ is the calculated outcoupling efficiency at the peak emission wavelength of $466 \mathrm{~nm}$ for $\operatorname{Ir}(\mathrm{dmp})_{3}, \varepsilon=3$ is the relative permittivity and $\epsilon_{0}$ is the vacuum permittivity. To account for the effect of water contamination during device fabrication ${ }^{26}, L(0)$ is normalized to 0.95 and $\Delta V(0)=0.2 \mathrm{~V}\left(\right.$ at $\left.3,000 \mathrm{~cd} \mathrm{~m}^{-2}\right)$ and $0.3 \mathrm{~V}\left(\right.$ at $\left.1,000 \mathrm{~cd} \mathrm{~m}^{-2}\right)$ for D1-D3, and $0.1 \mathrm{~V}\left(\right.$ at $3,000 \mathrm{~cd} \mathrm{~m}^{-2}$ ) and $0.15 \mathrm{~V}\left(\right.$ at $1,000 \mathrm{~cd} \mathrm{~m}^{-2}$ ) for D1S and D3S.

\section{References}

1. Tang, C. \& VanSlyke, S. Organic electroluminescent diodes. Appl. Phys. Lett. 51, 913 (1987).

2. National Research Council. Accessment of Advanced Solid-State Lighting (The National Academies Press, 2013).

3. Chung, H.-K. The challenges and opportunities of large OLED TVs. SID Inform. Display 29, 4 (2013).

4. Tsujimura, T. OLED Display Fundamentals and Applications Vol. 26 (Wiley.com, 2012).

5. Baldo, M. A., O'Brien, D. F., Thompson, M. E. \& Forrest, S. R. Excitonic singlet-triplet ratio in a semiconducting organic thin film. Phys. Rev. B 60, 14422 (1999).

6. Zhang, Y. \& Forrest, S. R. Triplets contribute to both an increase and loss in fluorescent yield in organic light emitting diodes. Phys. Rev. Lett. 108, 267404 (2012).

7. Kondakov, D. Y., Pawlik, T. D., Hatwar, T. K. \& Spindler, J. P. Triplet annihilation exceeding spin statistical limit in highly efficient fluorescent organic light-emitting diodes. J. Appl. Phys. 106, 124510 (2009).

8. Adachi, C., Baldo, M. A., Thompson, M. E. \& Forrest, S. R. Nearly $100 \%$ internal phosphorescence efficiency in an organic light-emitting device. J. Appl. Phys. 90, 5048 (2001).

9. Baldo, M. A. et al. Highly efficient phosphorescent emission from organic electroluminescent devices. Nature 395, 151-154 (1998).

10. Chwang, A. B., Kwong, R. C. \& Brown, J. J. Graded mixed-layer organic lightemitting devices. Appl. Phys. Lett. 80, 725-727 (2002).

11. Kwong, R. C. et al. High operational stability of electrophosphorescent devices. Appl. Phys. Lett. 81, 162-164 (2002).

12. Kim, S. H., Jang, J. \& Lee, J. Y. Lifetime improvement of green phosphorescent organic light-emitting diodes by charge confining device structure. Appl. Phys. Lett. 90, 203511 (2007).

13. Chin, B. D. \& Lee, C. Confinement of charge carriers and excitons in electrophosphorescent devices: mechanism of light emission and degradation. Adv. Mater. 19, 2061-2066 (2007).

14. Holmes, R. J. et al. Blue organic electrophosphorescence using exothermic hostguest energy transfer. Appl. Phys. Lett. 82, 2422-2424 (2003). 
15. Seifert, R. et al. Chemical degradation mechanisms of highly efficient blue phosphorescent emitters used for organic light emitting diodes. Org. Electron. 14, 115-123 (2013).

16. Giebink, N., DAndrade, B., Weaver, M., Brown, J. \& Forrest, S. Direct evidence for degradation of polaron excited states in organic light emitting diodes. J. Appl. Phys. 105, 124514 (2009).

17. Giebink, N. et al. Intrinsic luminance loss in phosphorescent small-molecule organic light emitting devices due to bimolecular annihilation reactions. J. Appl. Phys. 103, 044509 (2008).

18. Erickson, N. C. \& Holmes, R. J. Investigating the role of emissive layer architecture on the exciton recombination zone in organic light-emitting devices. Adv. Funct. Mater. 23, 5190-5198 (2013).

19. Chin, B. D., Lee, S.-H., Kim, J. K. \& Lee, C. H. Role of carrier mobility, exciton diffusion, and their interplay for charge balance and improved properties of organic electrophosphorescent device. Proc. SPIE 6333, Organic Light Emitting Materials and Devices X 633315, 15-19 (2006).

20. Liu, Z., Helander, M. G., Wang, Z. \& Lu, Z. Highly efficient two component phosphorescent organic light-emitting diodes based on direct hole injection into dopant and gradient doping. Org. Electron. 14, 852-857 (2013).

21. Lei, G., Wang, L. \& Qiu, Y. Improved performance of electrophosphorescent organic light-emitting diode by graded doped emissive layer. Jpn J. Appl. Phys. 43, L1226 (2004).

22. Forrest, S. et al. The stacked OLED (SOLED): a new type of organic device for achieving high-resolution full-color displays. Synth. Met. 91, 9-13 (1997).

23. Bulovic, V. et al. Weak microcavity effects in organic light-emitting devices. Phys. Rev. B 58, 3730-3740 (1998).

24. Kanno, H., Holmes, R. J., Sun, Y., Kena-Cohen, S. \& Forrest, S. R. White stacked electrophosphorescent organic light-emitting devices employing $\mathrm{MoO} 3$ as a charge-generation layer. Adv. Mater. 18, 339-342 (2006).

25. Celebi, K., Heidel, T. \& Baldo, M. Simplified calculation of dipole energy transport in a multilayer stack using dyadic Green's functions. Opt. Express 15, 1762-1772 (2007)

26. Yamamoto, H. et al. Improved initial drop in operational lifetime of blue phosphorescent organic light emitting device fabricated under ultra high vacuum condition. Appl. Phys. Lett. 99, 033301 (2011).

27. Wang, Q. \& Aziz, H. Degradation of organic/organic interfaces in organic lightemitting devices due to polaron-exciton interactions. ACS Appl. Mater. Interfaces 5, 8733-8739 (2013).
28. Féry, C., Racine, B., Vaufrey, D., Doyeux, H. \& Cina, S. Physical mechanism responsible for the stretched exponential decay behavior of aging organic light-emitting diodes. Appl. Phys. Lett. 87, 213502 (2005).

29. Xiang, C., Koo, W., So, F., Sasabe, H. \& Kido, J. A systematic study on efficiency enhancements in phosphorescent green, red and blue microcavity organic light emitting devices. Light Sci. Appl. 2, e74 (2013).

30. Anderson, M., Motta, R., Chandrasekar, S. \& Stokes, M. in Fourth Color Imaging Conference: Color Science, Systems, and Applications 238-245 (Society for Imaging Science and Technology, 1996).

31. Forrest, S. R., Bradley, D. D. C. \& Thompson, M. E. Measuring the efficiency of organic light-emitting devices. Adv. Mater. 15, 1043-1048 (2003).

\section{Acknowledgements}

We thank Anurag Panda for ultraviolet photoelectron spectra measurements, and Yue $\mathrm{Qu}$ for calculating the outcoupling characteristics. We acknowledge the U.S. Department of Energy, Center for Energy Nanoscience at the University of Southern California (DE-SC0001013) and Universal Display Corp. (UDC) for partial support of this work. We also thank UDC for providing materials and other experimental supplies, and Michael Slootsky for assistance in the lifetime measurement.

\section{Author contributions}

Y.Z. and S.R.F. conceived the experimental concept, analysed the data and prepared the manuscript. Y.Z. and J.L. fabricated and characterized the OLEDs.

\section{Additional information}

Supplementary Information accompanies this paper at http://www.nature.com/ naturecommunications

Competing financial interests: S.R.F. has a minor ownership interest in one of the sponsors of this work (UDC). All other authors declare no competing financial interest.

Reprints and permission information is available online at http://npg.nature.com/ reprintsandpermissions/

How to cite this article: Zhang, Y. et al. Tenfold increase in the lifetime of blue phosphorescent organic light-emitting diodes. Nat. Commun. 5:5008 doi: 10.1038/ ncomms6008 (2014). 